\title{
NEW BOUNDS ON THE NUMBER OF BOUND STATES FOR SCHRÓDINGER OPERATORS
}

\author{
K. Chadan \\ Laboratoire de Physique Théorique et Hautes Energies \\ Université de Paris XI, Bâtiment 211 \\ F - 91405 Orsay Cedex \\ and
}

A. Martin and J. Stubbe

Theoretical Physics Division, CERN

CH - 1211 Geneva 23

\begin{abstract}
We consider the Schrödinger operator $H=-\Delta+V(|x|)$ on $R^{3}$. Let $n_{\ell}$ denote the number of bound states with angular momentum $\ell$ (not counting the $2 \ell+1$ degeneracy). We prove the following bounds on $n_{\ell}$. Let $V \leq 0$ and $d / d r r^{1-2 p}(-V)^{1-p} \leq 0$ for some $p \in[1 / 2,1)$ then

$$
n_{\ell} \leq p(1-p)^{p-1}(2 \ell+1)^{1-2 p} \int_{0}^{\infty}\left(-r^{2} V\right)^{p} \frac{d r}{r} .
$$

This bound closes the gap between the celebrated bounds by Calogero $(p=1 / 2)$ and Bargmann $(p=1)$.
\end{abstract}

CERN-TH.7445/94

September 1994 


\section{Introduction}

A standard problem in the study of Schrödinger operators is to obtain bounds on the number of bound states in terms of integrals over the potential $V$. The most important bound which played an important rôle in the proof of the stability of matter is in terms of the classical phase space expression proved by Cwickel [1], Lieb [2] and Rosenblum [3] in the seventies

$$
N(V) \leq C \int_{\mathbf{R}^{3}}\left|V^{-}(x)\right|^{3 / 2} d^{3} x
$$

Here units are chosen such that $\hbar^{2} / 2 m=1$ where $V^{-}$denotes the attractive part of $V$, i.e., $V^{-}=\sup (-V, 0)$.

For spherically symmetric potentials there is the following bound by Bargmann [4]. If $n_{\ell}(V)$ denotes the number of bound states with angular momentum $\ell$ (not counting the $2 \ell+1$ degeneracy), then

$$
n_{\ell}(V) \leq(2 \ell+1)^{-1} \int_{0}^{\infty} V^{-}(r) r d r
$$

In 1965, Calogero [5] derived the following condition for monotonous purely attractive potentials

$$
n_{\ell}(V) \leq \frac{2}{\pi} \int_{0}^{\infty}\left|V^{-}(r)\right|^{1 / 2} d r
$$

Conditions on the non-existence of bound states have been derived by Glaser et al. [6] involving arbitrary powers $p \geq 1$ of the potential. More precisely, if

$$
\begin{aligned}
& C_{p} \frac{1}{4 \pi} \int_{R^{3}}|x|^{2 p-3}\left|V^{-}(x)\right|^{p} d^{3} x<1 \\
& C_{p} \equiv \frac{(p-1)^{p-1} \Gamma(2 p)}{p^{p} \Gamma^{2}(p)}, \quad p \geq 1,
\end{aligned}
$$

then there is no bound state, i.e., $N(V)=0$. This condition is valid only for spherically symmetric potentials if $1 \leq p<3 / 2$ and for arbitrary $V$ if $p \geq 3 / 2$. For spherically symmetric potentials the following conditions have been found in [6]. If, for some $p \geq 1$,

$$
(2 \ell+1)^{1-2 p} C_{p} \int_{0}^{\infty}\left(r^{2} V^{-}(r)\right)^{p} \frac{d r}{r}<1
$$

then $n_{\ell}(V)=0$. The constants $C_{p}$ are the best possible. An inequality on $n_{\ell}$ can be obtained by noticing that if there are $n_{\ell}$ bound states, the zero energy wave function regular at the origin has $n_{\ell}$ nodes, and by applying the converse of (5) to every nodal interval. In this way one gets

$$
n_{\ell}(V) \leq(2 \ell+1)^{1-2 p} C_{p} \int_{0}^{\infty}\left(r^{2} V^{-}(r)\right)^{p} \frac{d r}{r}
$$

The following bound on $N(V)$ for $p \geq 3 / 2$ corresponding to (4) has been proved by Blanchard et al. [7]

$$
N(V) \leq e^{p-1} C_{p} \frac{1}{4 \pi} \int_{R^{3}}|x|^{2 p-3}\left|V^{-}(x)\right|^{p} d^{3} x .
$$


Since the paper by Glaser et al. [6] there was the conjecture that for spherically symmetric potentials there should be bounds on $n_{\ell}(V)$ involving powers $p<1$ of the potential probably under some restrictions on $V$ since Calogero's bound which corresponds to $p=1 / 2$ only holds for monotonous potentials. The first attempt was made by one of us (K.C.) and R. Kobayashi [8]. If $V(r)$ is purely attractive and $d / d r\left(r^{1-2 p}(-V)^{1-p}\right) \leq 0$ for some $p \in[1 / 2,1]$ then there is a bound on $n_{\ell}(V)$ containing the powers $p$ and $1-p$. In particular at the two borders the bound tends to the bounds by Bargmann and Calogero, respectively.

However, the bound is not of the form one should expect. In the present paper we shall prove bounds which are the "natural" interpolations between the bounds by Calogero and Bargmann.

Theorem: Let $V$ be a spherically symmetric potential $V=V(r)$ satisfying

(i) $V \leq 0$;

(ii) $d / d r\left(r^{1-2 p}(-V)^{1-p}\right) \leq 0$ for some $p \in[1 / 2,1)$.

Then

$$
n_{\ell}(V) \leq p(1-p)^{p-1}(2 \ell+1)^{1-2 p} \int_{0}^{\infty}\left(-r^{2} V\right)^{p} \frac{d r}{r} .
$$

Notice that condition (ii) is weaker than the requirement of monotonicity of the Calogero bound.

\section{Proof of the main result}

First of all we prove that a bound for $\ell=0$ implies a bound for $\ell>0$.

Lemma 1: Let $V$ satisfy the conditions of the theorem. If there is $\tilde{C}_{p}>0$ such that $n_{0}(V) \leq$ $\tilde{C}_{p} \int_{0}^{\infty}\left(r^{2} V\right)^{p} d r / r, 1 / 2 \leq p \leq 1$, then $n_{\ell}(V) \leq \tilde{C}_{p}(2 \ell+1)^{1-2 p} \int_{0}^{\infty}\left(-r^{2} V\right)^{p} d r / r$

Proof: Consider the reduced hamiltonians

$$
h_{\ell}=-\frac{d^{2}}{d r^{2}}+\frac{\ell(\ell+1)}{r^{2}}+V(r) \text { on }(0, \infty)
$$

It is a well-known result that $n_{\ell}(V)$ equals the number of nodes of the zero energy solution in $(0, \infty)$ satisfying Dirichlet boundary conditions at the origin. Let $u_{\ell}$ be the solution of $h_{\ell} u_{\ell}=0$.

Define $s=s(r)$ and $\varphi_{\ell}(s)$ by

$$
s(r)=\frac{r^{2 \ell+1}}{2 \ell+1}, \quad \varphi_{\ell}(s)=r^{\ell} u_{\ell}(r)
$$

Then $\varphi_{\ell}(s)$ satisfies

$$
-\frac{d^{2}}{d s^{2}} \varphi_{\ell}(s)+\tilde{V}(s) \varphi_{\ell}(s)=0
$$

with

$$
\tilde{V}(s)=\frac{V(r(s))}{r(s)^{4 \ell}} \quad \text { and } \quad \varphi_{\ell}(0)=0
$$


Obviously, the number of nodes of $u_{\ell}$ and $\varphi_{\ell}$ in $(0, \infty)$ coincides. $\tilde{V}(s)$ satisfies the same conditions as $V(r)$ since $\tilde{V}(s) \leq 0$ and

$$
\begin{aligned}
\frac{d}{d s} s^{1-2 p}(-\tilde{V}(s))^{1-p} & =r^{-2 \ell} \frac{d}{d r}\left(r^{-2 \ell / 1-p} r^{1-2 p}\left(-V(r)^{1-p}\right)\right. \\
& \leq 0
\end{aligned}
$$

Therefore

$$
n_{\ell}(v) \leq \tilde{C}_{p} \int_{0}^{\infty}\left(-s^{2} V^{2}(s)\right)^{p} \frac{d s}{s}
$$

Since the right-hand side of (12) equals

$$
(2 \ell+1)^{1-2 p} \tilde{C}_{p} \int_{0}^{\infty}\left(-r^{2} V(r)\right)^{p} \frac{d r}{r}
$$

the lemma is proved.

For $\ell=0$ we need the following properties of $V$.

Lemma 2: Let $I(R)=\int_{0}^{R}\left(-r^{2} V\right)^{p} d r / r$. If $d / d r\left(r^{1-2 p}(-V)^{1-p}\right)<0$, then

$$
I(R) \geq \frac{1-p}{p}\left(-r^{2} V(R)\right)^{p}
$$

Proof: We write $I(R)$ as

$$
I(R)=\int_{0}^{R}\left(-V(r) r^{1-2 p / 1-p}\right)^{p} r^{(2 p-1) /(1-p)} d r .
$$

The first term in the integral is decreasing since $p<1$ and may be bounded below by $\left(-V(R) R^{1-2 p / 1-p}\right)^{p}$. Integrating the second term we obtain the desired inequality.

Lemma 3: If $V \leq 0$ and $d / d r\left(r^{1-2 p}(-V)^{1-p}\right) \leq 0$ (for some $p \in[-1 / 2,1)$ then

$$
\int_{0}^{\infty}\left(-r^{2}(V(r)) \frac{d r}{r}<\left[\tilde{C}_{p} \int_{0}^{\infty}\left(-r^{2} V(r)\right)^{p} \frac{d r}{r}\right]^{1 / p}\right.
$$

where $\tilde{C}_{p}=p(1-p)^{p-1}$

Proof: We have

$$
\int_{0}^{\infty}(-r V) d r=\int_{0}^{\infty} \frac{d I(r)}{d r}\left(-r^{2} V\right)^{1-p} d r
$$

By Lemma 2,

$$
\int_{0}^{\infty}(-r V) d r<\int_{0}^{\infty} \frac{d I(r)}{d r}\left[\frac{p}{1-p} I(r)\right]^{(1-p) / p}
$$

Since

$$
\frac{d I(r)}{d r} I(r)^{\frac{1-p}{p}}=p \frac{d}{d r}\left(I(r)^{1 / p}\right)
$$


the result follows.

Now we are in position to prove the bound for $\ell=0$.

If the zero energy solution for $h_{0}$ has $n_{0}(V)$ zeros then by Bargmann's bound

$$
n_{0}(V)<\int_{0}^{\infty}(-r V(r)) d r
$$

In particular (if there is one bound state) then by Lemma 3

$$
\tilde{C}_{p} \int_{0}^{\infty}\left(-r^{2} V(r)\right)^{p} \frac{d r}{r}>1
$$

Now, consider the interval $\left[r_{k}, r_{k+1}\right]$ between two successive nodes of the zero energy solution. We define $V_{k}(r)$ by

$$
V_{k}(r)=\left\{\begin{array}{cc}
V(r) & r \leq r_{k+1} \\
0 & r>r_{k+1}
\end{array}\right.
$$

Then $V_{k}(r)$ satisfies the same conditions as $V(r)$. Changing the origin $x=r-r_{k}$ we consider $V_{k}\left(x+r_{k}\right)$ for $x \geq 0$. Since $d / d r\left(r^{1-2 p}\left(-V(r)^{1-p}\right) \leq 0\right.$ reads

$$
(1-p) \frac{(-d V / d r)}{(-V)}<\frac{2 p-1}{r}
$$

we also have

$$
(1-p) \frac{-d V / d r}{(-V)}<\frac{2 p-1}{r-r_{k}} \quad \text { for } \quad r>r_{k}
$$

since $p \geq 1 / 2$.

Hence, by Eq. (14)

$$
\begin{aligned}
1 & <\tilde{C}_{p} \int_{0}^{\infty}\left(-V_{k}\left(x+r_{k}\right)\right)^{p} x^{2 p-1} d x \\
& =\tilde{C}_{p} \int_{r_{k}}^{r_{k+1}}(-V(r))^{p}\left(r-r_{k}\right)^{2 p-1} d r \\
& <\tilde{C}_{p} \int_{r_{k}}^{r_{k+1}}(-V(r))^{p} r^{2 p-1} d r
\end{aligned}
$$

Consequently, if we add up all contributions we find

$$
n_{0}(V)<\tilde{C}_{p} \int_{0}^{\infty}\left(-r^{2} V(r)\right)^{p} \frac{d r}{r}
$$

\section{Conclusions and a conjecture about the optimal con- stants}

We have proved bounds on the number of bound states in a spherically symmetric potential which interpolate between the bounds by Calogero and Bargmann. In particular, a simple 
"transformation of variables" argument showed that a bound on the zero angular momentum sector implies a (non-trivial) bound for non-zero angular momenta.

The constants $\tilde{C}_{p}=p(1-p)^{p-1}$ are not optimal for $p<1$ but we suppose that they are not too far from the optimal values since as $p \rightarrow 1, \tilde{C}_{p}$ tends to the value in Bargmann's bound which is known to be optimal and for $p=1 / 2$ we have $c_{1 / 2}=1 / 2 \sqrt{2}=0.707, \ldots$ which is not too far from $2 / \pi=0.6366 \ldots$

We conjecture that the optimal constants (at least for $\ell=0$ ) are related to the potentials which saturate the first-order differential inequality on $V$ as follows.

Let $V_{p}(r)$ be given by

$$
V_{p}(r)=\left\{\begin{array}{cc}
-r^{2 p-1 / 1-p} & 0 \leq r \leq R \\
0 & r>R
\end{array}\right.
$$

for some $R>0$. $V_{p}(r)$ satisfies $d / d r\left(r^{1-2 p}(-V)^{(-p)}=0\right.$ in $(0, R)$ and in $(R, \infty)$.

The zero energy solution in $(0, R)$ is

$$
u_{p}(r)=r^{1 / 2} J_{1-p}\left(2(1-p) r^{1 / 2(1-p)}\right)
$$

where $J_{1-p}($.$) denotes the standard Bessel function.$

If we choose $R=R_{p}$ as the first critical point of $u_{p}(r)$ given in (21), then $u_{p}$ corresonds to a zero energy eigenstates. We conjecture that

$$
\begin{aligned}
C_{p} & \equiv \frac{1}{\int_{0}^{R_{p}}\left(-r^{2} V_{p}\right)^{p \frac{d r}{r}}} \\
& =\frac{p}{1-p} R_{p}^{-p / 1-p}
\end{aligned}
$$

is the optimal constant (which is true as $p \rightarrow 1$ and for $p=1 / 2$ ).

A more explicit condition for $R_{p}$ can be obtained as follows.

Defining the change of variables

$$
z=2(1-p) r^{1 / 2(1-p)}
$$

and defining the function $\varphi_{p}(z)$ by

$$
\varphi_{p}(z)=z^{1-p} J_{1-p}(z)
$$

we compute that

$$
z_{p} \equiv 2(1-p)\left(R_{p}\right)^{1 / 2(p-1)}
$$

is the first critical point of $\phi_{p}$.

Since $d / d z \varphi_{p}(z)=z^{1-p} J_{-p}(z)$ we see that $z_{p}$ is the first zero of $J_{-p}(z)$ and

$$
C_{p}=p(1-p)^{2 p-1}\left(\frac{z_{p}}{2}\right)^{-2 p}
$$


In the following Table we list some numerical values for $C_{p}$ and compare them with our constant $\tilde{C}_{p}=p(1-p)^{p-1}$.

\begin{tabular}{|c|c|c|c|}
\hline$p$ & $\tilde{C}_{p}$ & $C_{p}$ & $\frac{C_{p}}{C_{p}}$ \\
\hline 0.50 & 0.7071 & 0.6366 & 1.111 \\
0.55 & 0.7878 & 0.7089 & 1.111 \\
0.60 & 0.8656 & 0.7802 & 1.110 \\
0.65 & 0.9386 & 0.8492 & 1.105 \\
0.70 & 1.0045 & 0.9145 & 1.099 \\
0.75 & 1.0607 & 0.9739 & 1.089 \\
0.80 & 1.1038 & 1.0250 & 1.077 \\
0.85 & 1.1298 & 1.0639 & 1.062 \\
0.90 & 1.1330 & 1.0851 & 1.044 \\
0.95 & 1.1035 & 1.0781 & 1.024 \\
1.00 & 1.0000 & 1.0000 & 1.000 \\
\hline
\end{tabular}

We conclude that our bound is not far from being optimal, especially for $p$ close to unity.

\section{References}

[1] M. Cwickel, Ann.Math. 106 (1977) 93.

[2] E. Lieb, Bull.Am.Math.Soc. 82 (1976) 751; in Proc.Am.Math.Soc. Symposium in Pure Mathematics, eds. R. Ossermann and A. Weinstein, Vol.36 (1980) 241.

[3] G.V. Rosenbljum, Dokl.Akad.Nauk.SSSR 202 (1012 (1972).

[4] V. Bargmann, Proc.Nat.Acad.Sci.(US) 39 (1952) 961.

[5] F. Calogero, Commun.Math.Phys. 1 (1965) 80.

[6] V. Glaser, H. Grosse, A. Martin and W. Thirring, in Studies in Mathematical PHysics, eds. E. Lieb, B. Simon, A. Wightman, Princeton University PRess 1976, pp. 169-194.

[7] Ph. Blanchard, J. Stubbe and G. Rezende, Lett.Math.Phys. 14 (1987) 215.

[8] K. Chadan and R. Kobayashi, in Rigorous Methods in Particle PHysics, eds. S. Ciulli, F. Scheck and W. Thirring, Springer-Berlin (1990), p. 14. 